\title{
Cobertura da Estratégia Saúde da Família no Brasil: o que nos mostram as Pesquisas Nacionais de Saúde 2013 e 2019
}

\author{
The Family Health Strategy coverage in Brazil: \\ what reveal the 2013 and 2019 National Health Surveys
}

Ligia Giovanella (https://orcid.org/0000-0002-6522-545X) ${ }^{1}$

Aylene Bousquat (https://orcid.org/0000-0003-2701-1570) ${ }^{2}$

Simone Schenkman (https://orcid.org/0000-0003-1140-1056) ${ }^{2}$

Patty Fidelis de Almeida (https://orcid.org/0000-0003-1676-3574) ${ }^{3}$

Luciana Monteiro Vasconcelos Sardinha (https://orcid.org/0000-0002-3679-3618) ${ }^{4}$

Maria Lúcia França Pontes Vieira (https://orcid.org/0000-0002-5284-6214) ${ }^{5}$

${ }^{1}$ Escola Nacional de Saúde Pública Sérgio Arouca, Fundação Oswaldo Cruz. R. Leopoldo Bulhões 1480, Manguinhos. 21041-210 Rio de Janeiro RJ Brasil.

ligiagiovanella@gmail.com

${ }^{2}$ Faculdade de Saúde

Pública, Universidade de

São Paulo. São Paulo SP

Brasil.

${ }^{3}$ Instituto de Saúde Coletiva,

Universidade Federal

Fluminense. Niterói RJ

Brasil.

${ }^{4}$ Departamento de

Análise em Saúde e

Vigilância de Doenças Não Transmissíveis, Secretaria

de Vigilância em Saúde, Ministério da Saúde. Brasília DF Brasil.

${ }^{5}$ Diretoria de Pesquisas, Instituto Brasileiro de

Geografia e Estatística. Rio de Janeiro RJ Brasil.
Abstract This paper examines the evolution of Brazil's Family Health Strategy coverage from the findings of the 2013 and 2019 National Health Survey censuses. Indicators included Family Health Clinic coverage of residents and households, frequency of visits by Community Health Workers, and usual source of care, all stratified by rural and urban areas, Brazilian regions, states, education of the household head, and income quintile. In 2019, 60.0\% of households were enrolled in a Family Health Clinic, and population coverage was $62.6 \%$. Coverage was higher in rural than in urban areas in the Northeast and South regions. Between 2013 and 2019, coverage increased by $11.6 \%$, while monthly health worker visits decreased. Coverage was highest among the most vulnerable population, as defined by the household head education level or by the family income. Availability of usual source of care was highest among those enrolled in a Family Health Clinic. The 2019 National Health Survey findings confirm that Brazil's Family Health Strategy continues to be an equitable policy and the main SUS' Primary Health Care model. However, recent changes in the national policy guidance, which are weakening the community approach and the priority given to the Family Health Strategy Program, may jeopardize those gains.

Key words Primary Health Care, Family Health Strategy, Health surveys, Health services coverage, Access
Resumo $O$ artigo analisa a evolução da cobertura da Estratégia de Saúde da Família (ESF), a partir dos resultados dos inquéritos populacionais das Pesquisas Nacionais de Saúde (PNS) de 2013 e 2019. Foram calculados indicadores de cobertura de moradores e domicílios por Unidade de Saúde da Família (USF), frequência da visita de Agente Comunitário de Saúde (ACS), serviço de procura regular e tipo de serviço buscado; estratificados por área rural e urbana, grandes regiões, unidades da federação, escolaridade do responsável pelo domicílio e quintis de renda. Em 2019, 60,0\% dos domicílios estavam cadastrados em USF e a cobertura de moradores era $62,6 \%$. A cobertura é superior na área rural e nas regiões Nordeste e Sul. Entre 2013 e 2019, observa-se aumento de cobertura em 11,6\% e redução na visita mensal do ACS. A cobertura é mais elevada entre a população mais vulnerável, considerada escolaridade do responsável pelo domicílio ou renda familiar. A disponibilidade de serviço de procura regular é maior entre cadastrados na USF. Os resultados da PNS 2019 reiteram que a ESF permaneceu como política equitativa $e$ principal modelo de APS no SUS. No entanto, as recentes mudanças na condução da política nacional, que enfraquecem o enfoque comunitário e a prioridade da ESF, podem ameaçar tais avanços. Palavras-chave Atenção Primária à Saúde, Estratégia de Saúde da Família, Inquéritos epidemiológicos, Cobertura de serviços de saúde, Acesso 


\section{Introdução}

Sistemas de saúde orientados por uma Atenção Primária à Saúde (APS), centrada nas pessoas e comunidades e capaz de responder às diferentes necessidades de saúde dos grupos populacionais, têm sido associados a inúmeros resultados positivos em saúde. Pacientes com uma ou mais condições de cronicidade, por exemplo, apresentam melhor autopercepção de saúde em países com forte estrutura de APS, com continuidade e oferta abrangente de serviços em sistemas públicos de saúde ${ }^{1}$. No Brasil, resultados positivos em saúde foram alcançados com a expansão da Estratégia Saúde da Família (ESF), modelo brasileiro de atenção primária à saúde de orientação comunitária, com destaque para a redução da mortalidade infantil, redução de internações por condições sensíveis à atenção primária e redução da mortalidade por doenças cardiovasculares ${ }^{2-4}$. A ESF se consolidou, a partir dos anos 2000, como a principal política de APS no país, fortemente ancorada nos atributos essenciais e derivados ${ }^{5}$ e nas dimensões de participação social, intersetorialidade e multidisciplinaridade, alinhadas aos princípios de universalidade, integralidade e equidade do Sistema Único de Saúde (SUS).

Dados do Suplemento Saúde da PNAD 2008 já indicavam que $50,9 \%$ da população brasileira, estava coberta pela ESF, com aumento na cobertura para 53,4\% em 2013, segundo a Pesquisa Nacional de Saúde (PNS) de 2013, resultados coincidentes com as estimativas a partir dos dados administrativos do Ministério da Saúde à época ${ }^{6}$. Não menos importante, foi a ampliação da referência a um serviço de busca regular pela grande maioria da população (78\%), embora se mantivessem diferenças regionais e por nível de instrução, de acordo com a PNS 20137 . Desigualdades que expressam as contradições e condicionantes da política de saúde brasileira e limitam a consolidação de um sistema de saúde público universal.

Acompanhar a evolução da cobertura dos serviços de saúde em geral, e de APS em particular, é essencial para se identificar algumas das barreiras de acesso aos serviços de saúde e contribuir para informar a formulação de políticas de saúde. Neste sentindo é imprescindível aumentar os esforços para a padronização de instrumentos, a melhoria da qualidade e disponibilidade de dados para medir a cobertura de determinada intervenção e as desigualdades de acesso, inclusive em uma perspectiva regional que permita, entre outros aspectos, a comparabilidade entre os países 9 . No Brasil, o monitoramento de indicadores de acesso e uso de serviços de saúde tem sido realizado por meio de pesquisas de base populacional como a PNS que se apresenta como um instrumento imprescindível para a avaliação e orientar a qualificação do SUS, sobretudo em ambiente de acirrado e sistemático ataque às políticas sociais.

No país, após um período de acelerada expansão da ESF, a partir dos anos de 2010, políticas nacionais buscaram induzir e institucionalizar o apoio multiprofissional, processos de avaliação do acesso e da qualidade, a melhoria da infraestrutura das Unidades Básicas de Saúde (UBS) e a provisão de médicos para a atenção básica, com resultados distintos a depender do contexto de implantação ${ }^{10}$. No caso da ESF, a PNS 2019 é a primeira pesquisa de base populacional nacional realizada após as mudanças propostas pela Política Nacional de Atenção Básica (PNAB) de 2017 e por um conjunto de leis e normativas que instituem nova modalidade de financiamento, carteira de serviços e composição das equipes. Estas medidas, que contemplam forte indução para o estabelecimento de relações entre o Estado e empresas privadas para a gestão e provisão de serviços, e enfraquecem a abordagem territorial, o trabalho comunitário, o cuidado integral e multidisciplinar $^{11}$, exigirão um acompanhamento atento de toda sociedade brasileira.

A PNS de 2019 coletou dados sobre acesso e utilização de serviços de saúde em amostra nacional de âmbito domiciliar, com o objetivo de identificar a situação de saúde e os estilos de vida da população brasileira e obter informações sobre a atenção à saúde, no que se refere ao acesso e uso dos serviços, à continuidade dos cuidados e ao financiamento da assistência ${ }^{12}$. O objetivo deste artigo é analisar a evolução da cobertura da ESF a partir dos resultados dos inquéritos populacionais das PNS, entre os anos de 2013 e 2019, contextualizando seus resultados frente às recentes mudanças na condução da política nacional de atenção básica no país.

\section{Metodologia}

O artigo analisa a evolução da cobertura da ESF ente 2013 e 2019, estimada nacionalmente, para áreas urbana e rural, pelas grandes regiões nacionais e unidades da federação (UF).

A PNS é uma pesquisa nacional, que tem por população-alvo, a população residente nos domicílios particulares de áreas rurais e urbanas 
brasileiras, sendo realizada em uma amostra probabilística de domicílios. Resulta de uma parceria do Ministério da Saúde (MS) com o Instituto Brasileiro de Geografia e Estatística (IBGE) e faz parte do Sistema Integrado de Pesquisas Domiciliares (SIPD) do IBGE. Utiliza uma subamostra da Amostra Mestra do SIPD com a mesma estratificação das unidades primárias de amostragem (UPA), constituídas por um ou mais setores censitários.

O tamanho da amostra da PNS 2019 foi definido em 8.036 UPA com 108.525 domicílios, considerando-se uma taxa de não resposta de $20 \%{ }^{12}$.

O plano amostral da PNS foi por conglomerados divididos em três estágios. No primeiro foram selecionadas as UPA (setores censitários ou conjunto de setores), com probabilidade proporcional ao tamanho, definida pelo número de domicílios particulares permanentes, e a seleção para a amostra da PNS, com probabilidade igualmente proporcional. A distribuição da quantidade de domicílios a serem entrevistados por UPA variou de 12 a 18 domicílios e é inversamente proporcional à quantidade de domicílios por UF. Assim, no Maranhão, Ceará, Pernambuco, Bahia, Minas Gerais, Rio de Janeiro, São Paulo, Paraná, Santa Catarina e Rio Grande do Sul, foram sorteados 12 domicílios por UPA. Em Rondônia, Acre, Amazonas, Pará, Piaú, Rio Grande do Norte, Paraíba, Alagoas, Sergipe, Espírito Santo, Mato Grosso do Sul, Mato Grosso, Goiás e Distrito Federal este número foi de 15. Em Roraima, Amapá e Tocantins foram selecionados 18 por UPA. Em cada domicílio amostrado foi selecionado um morador com 15 anos ou mais. O método de seleção em todos os estágios foi a amostra aleatória simples.

O questionário continha três partes: i) para a coleta de dados referentes ao domicílio e visitas domiciliares realizadas por agentes de endemias e equipes de Saúde da Família; ii) para todos os moradores do domicílio, com enfoque na coleta de informações socioeconômicas, de saúde e de uso de serviços de saúde; e iii) para um morador selecionado foram aprofundados temas relacionados a estilos de vida, trabalho, doenças crônicas, violências, entre outros ${ }^{12,13}$.

Para as duas primeiras partes do questionário, as informações foram prestadas por uma pessoa moradora, considerada apta a prestar informação para o conjunto de moradores e sobre o domicílio. A terceira parte foi respondida pelo morador aleatoriamente selecionado. A técnica de coleta foi uma entrevista pessoal assistida por computador. A coleta de dados ocorreu entre os meses de agosto de 2019 e março de $2020^{12}$. Foram obtidas informações em 108.457 domicílios.

Neste estudo, os bancos utilizados foram fornecidos pelo IBGE, e já incluíam o cálculo do peso tanto para moradores quanto para domicílios, a ser considerado em amostra complexa. Para garantir a comparabilidade dos resultados das duas edições da pesquisa, o IBGE recalculou os fatores de expansão da PNS 2013, considerando a revisão da Projeção da População das Unidades da Federação, por sexo e idade, para o período 2010-2060, pelo Método das Componentes Demográficas. Ou seja, o IBGE fez a reponderação dos pesos utilizados para a expansão da amostra da PNS $2013^{12}$.

O software utilizado foi o IBM SPSS 22.0, os efeitos da estratificação e da conglomeração na estimação dos indicadores e suas medidas de precisão foram considerados.

Foram calculados os seguintes indicadores para as duas edições da PNS:

- \% de domicílios cadastrados em Unidades de Saúde da Família (USF),

- \% de moradores de domicílios cadastrados em USF,

- \% de domicílios cadastrados na USF por frequência da visita do Agente Comunitário de Saúde (ACS),

- \% de moradores em domicílios cadastrados e não cadastrados na USF que referiam serviços de saúde de procura regular,

- \% de moradores em domicílios cadastrados e não cadastrados, que informaram serviço de procura regular, por tipo de serviço buscado.

Estes indicadores foram estratificados por: área rural ou urbana; grandes regiões; unidades da Federação; escolaridade do responsável do domicílio; e quintis de renda familiar per capita.

Foram descritas as prevalências e estimados os intervalos de confiança (IC95\%) para os dados da PNS 2019. Para as comparações entre os resultados da PNS 2013 e a PNS 2019 foi utilizado o teste de qui quadrado de Pearson ajustado; o nível de significância adotado foi 5\%.

\section{Resultados}

A PNS permite analisar a cobertura por USF segundo a proporção de domicílios cobertos e pela proporção de população em base ao número de moradores residentes nestes domicílios.

Em 2019, no Brasil 60,0\% (IC95\%: 58,9-61,0) dos informantes referiram que seu domicílio era 
cadastrado em Unidade de Saúde da Família (USF) e 11,0\% (IC95\% 10,5-11,6) não sabiam responder a esta pergunta (valor similar a 2013: $10,6 \%)$. A cobertura em domicílios de área rural $(77,0 \%)$ é superior à cobertura de domicílios em áreas urbanas $(57,3 \%)$. As regiões com maior cobertura de domicílios são o Nordeste $(71,1 \%)$ e o Sul $(64,8 \%)$. O Sudeste apresentou a mais baixa cobertura, $51,9 \%$, ainda que compreenda o maior número absoluto de domicílios cadastrados (16,6 milhões) (Tabela 1).

A cobertura, tomando como base o número de moradores no conjunto dos domicílios aumenta para 62,6\% (IC95\%: 61,5-63,7), um contingente expressivo de 131,2 milhões de pessoas. As diferenças observadas entre as regiões na cobertura por domicílios se mantêm para o denominador de moradores. Cinco estados têm cobertura populacional por USF maior de $80 \%$ são eles: Piauí (90,7\%), Tocantins (89,7\%), Santa Catarina $(87,2 \%)$, Paraíba $(86,7 \%)$ e Sergipe $(82,6 \%)$. Onze estados têm cobertura de mais de $70 \%$ de sua população. Em termos absolutos, São Paulo (21,9 milhões) e Minas Gerais (15,5 milhões) concentram $28,5 \%$ do total de moradores cadastrados em USF no país (Tabela 1).

$\mathrm{Na}$ Tabela 2, pode-se visualizar a evolução da cobertura populacional entre os anos de 2013 e 2019. No país como um todo observa-se aumento na cobertura de 6,5 pontos percentuais (pp), passando de $56,1 \%$ em 2013 para $62,6 \%$ da população em 2019, o que corresponde a um aumento proporcional de $11,6 \%$ em seis anos e a inclusão de mais 18,7 milhões de moradores na ESF.

Em todas as regiões do país os aumentos foram significativos, bem como nos domicílios urbanos e rurais. Os maiores aumentos na cobertura populacional ocorreram nas regiões Sul e Norte, 9,1 e 8,8 pp respectivamente. No entanto, quando a análise é realizada por Unidade da Federação observam-se comportamentos variados. O Pará e o Distrito Federal experimentaram percentuais elevados de crescimento, na faixa dos 16 pp. O Rio de Janeiro, Paraná, São Paulo, Santa Catarina, Mato Grosso, Amapá, Paraíba, Pernambuco, Piauí, Sergipe e Ceará também apresentaram crescimento significativo. As variações não foram significativas nos estados do Acre, Amazonas, Roraima, Maranhão, Rio Grande do Norte, Alagoas, Bahia, Minas Gerais, Espírito Santo, Rio Grande do Sul, Mato Grosso do Sul e Goiás. Em Tocantins que já tinha cobertura maior de $90 \%$ em 2013, observou-se leve redução (Tabela 2).

Quando analisada a variação percentual entre as duas pesquisas (penúltima coluna da Tabela
2), os aumentos de cobertura no Distrito Federal (112,3\%), Rio de Janeiro (38,2\%) e Pará $(35,8 \%)$ são ainda mais expressivos.

Um dos elementos centrais que caracterizam a ESF é a visita domiciliar (VD) de ACS às famílias sob sua responsabilidade, o que deve ser realizado rotineiramente, em função das necessidades e demandas das famílias e territórios. Considerando-se os domicílios cadastrados há mais de um ano, observou-se redução nas visitas de ACS entre 2013 e 2019 (Tabela 3). A proporção de domicílios que referem visita mensal do ACS no último ano, passou de 47,2\% (IC95\%: 45,7-48,8) em 2013 para 38,4\% (IC95\%: 37,4-39,4) em 2019. Observa-se também o aumento da proporção dos que nunca receberam visita domiciliar de ACS nos 12 meses anteriores à pesquisa, de 17,7\% (IC95\%: 16,6-18,8) em 2013 para 23,8\% (IC95\%: 22,924,7) em 2019. Os domicílios rurais cadastrados recebem visita do ACS com maior frequência do que os domicílios urbanos. Em 2019, 66,3\% dos domicílios rurais receberam visita mensal ou a cada dois meses, enquanto nos domicílios urbanos este percentual foi de $44,9 \%$ (Tabela 3 ).

Vale ressaltar, que considerada a evolução do número absoluto de domicílios que receberam visita de ACS mensalmente ou a cada dois meses nas duas PNS, observa-se acréscimo de 2,1 milhões de domicílios que passaram a receber visitas mensalmente, bem como acréscimo de 4,2 milhões de domicílios que embora cadastrados não receberam visita domiciliar em 2019.

A redução da proporção das visitas mensais do ACS foi observada para o Brasil como um todo, em todas as grandes regiões e nas áreas urbanas e rurais. A redução não é constante em todas as UF. Em 16 estados a redução de visita mensal é significativa (Tabela 3). Por sua vez, o aumento da proporção, em 2019, dos que nunca receberam visita domiciliar de ACS nos 12 meses anteriores à pesquisa foi também significativo em todas as Regiões e em 15 UF. Em oito estados não se observou diferença significativa, seja nas visitas mensais, seja em nenhuma visita no período (AM, AP, MA, AL, SE, BA, SP, MT) (Tabela 3).

Não obstante, em 2019, em oito estados (TO, MA, PB, AL, SC, MT, MS e GO) $60 \%$ ou mais dos domicílios receberam visita de ACS, mensal ou a cada dois meses.

Chama atenção que nas duas UF, Rio de Janeiro e Distrito Federal, que tiveram os maiores aumentos proporcionais na cobertura da ESF, a metade ou mais dos domicílios não tenham recebido uma VD de ACS e o percentual de domicílios que referem visitas mensais, em 2019, seja de 
Tabela 1. Proporção de domicílios cadastrados em unidades de saúde da família e de pessoas moradoras nos domicílios cadastrados, Brasil, Grandes Regiões, Unidades da Federação e situação do domicílio urbano/rural, 2019.

\begin{tabular}{|c|c|c|c|c|c|c|}
\hline & \multicolumn{3}{|c|}{$\begin{array}{c}\text { Domicílios cadastrados } \\
\text { na USF }(1.000)\end{array}$} & \multicolumn{3}{|c|}{$\begin{array}{l}\text { Moradores em domicílios } \\
\text { cadastrados na USF (1.000) }\end{array}$} \\
\hline & $\%$ & IC 95\% & $\mathbf{n}$ & $\%$ & IC 95\% & $\mathbf{n}$ \\
\hline Brasil & 60,0 & $58,9-61,0$ & 43.900 & 62,6 & $61,5-63,7$ & 131.201 \\
\hline Urbano & 57,3 & $56,1-58,5$ & 36.177 & 59,8 & $58,6-61,0$ & 107.092 \\
\hline Rural & 77,0 & $75,4-78,5$ & 7.723 & 78,9 & $77,3-80,4$ & 24.108 \\
\hline Norte & 60,0 & $58,1-61,9$ & 3.251 & 62,2 & $60,2-64,2$ & 11.278 \\
\hline Rondônia & 51,1 & $46,9-55,4$ & 301 & 52,8 & $48,6-56,9$ & 933 \\
\hline Acre & 52,2 & $48,6-58,2$ & 140 & 56,1 & $52,0-60,0$ & 487 \\
\hline Amazonas & 54.8 & $51,3-58,4$ & 611 & 58,5 & $54,6-62,3$ & 2.333 \\
\hline Roraima & 52,0 & $47,4-56,1$ & 79 & 54,0 & $48,4-58,3$ & 289 \\
\hline Pará & 61,3 & $57,8-64,7$ & 1.569 & 63,7 & $60,0-67,2$ & 5.455 \\
\hline Amapá & 43,3 & $37,2-50,0$ & 95 & 45,3 & $38,5-52,5$ & 380 \\
\hline Tocantins & 88,5 & $86,0-90,6$ & 457 & 89,7 & $87,2-91,7$ & 1.400 \\
\hline Nordeste & 71,1 & $70,0-72,3$ & 13.535 & 73,3 & $72,1-74,5$ & 41.751 \\
\hline Maranhão & 65,8 & $63,5-68,1$ & 1.389 & 68,4 & $65,9-70,8$ & 4.817 \\
\hline Piauí & 89,5 & $87,7-91,3$ & 941 & 90,7 & $88,7-92,3$ & 2.968 \\
\hline Ceará & 72,4 & $70,2-74,6$ & 2.170 & 73,8 & $71,6-75,9$ & 6.751 \\
\hline Rio Grande do Norte & 68,8 & $65,0-72,4$ & 774 & 71,1 & $67,4-74,5$ & 2.495 \\
\hline Paraíba & 85,3 & $82,4-87,8$ & 1.134 & 86,7 & $83,9-89,0$ & 3.464 \\
\hline Pernambuco & 70,6 & $67,4-73,7$ & 2.324 & 73,4 & $70,0-76,5$ & 6.974 \\
\hline Alagoas & 65,4 & $62,7-68,4$ & 710 & 67,8 & $64,8-70,6$ & 2.259 \\
\hline Sergipe & 80,3 & $77,9-82,9$ & 637 & 82,6 & $79,8-65,0$ & 1.900 \\
\hline Bahia & 65,9 & $62,8-68,9$ & 3.455 & 68,1 & $64,8-71,3$ & 10.123 \\
\hline Sudeste & 51,9 & $49,7-54,0$ & 16.594 & 54,6 & $52,3-56,8$ & 48.230 \\
\hline Minas Gerais & 70,3 & $66,3-74,0$ & 5.398 & 73,0 & $68,9-76,8$ & 15.456 \\
\hline Espírito Santo & 60,5 & $56,7-64,1$ & 859 & 61,7 & $57,7-65,5$ & 2.479 \\
\hline Rio de Janeiro & 45,5 & $42,8-48,2$ & 2.948 & 48,5 & $45,6-51,5$ & 8.380 \\
\hline São Paulo & 45,0 & $41,5-48,6$ & 7.389 & 47,7 & $44,0-51,5$ & 21,914 \\
\hline Sul & 64,8 & $62,7-66,9$ & 7.201 & 67,6 & $65,4-69,7$ & 20.232 \\
\hline Paraná & 66,3 & $62,6-69,9$ & 2.738 & 68,5 & $64,7-72,2$ & 7.831 \\
\hline Santa Catarina & 85,0 & $82,2-87,5$ & 2.240 & 87,2 & $64,6-89,4$ & 6.241 \\
\hline Rio Grande do Sul & 51,2 & $47,4-55,0$ & 2.223 & 54,2 & $50,3-58,0$ & 6.160 \\
\hline Centro-Oeste & 58,6 & $56,4-60,8$ & 3.311 & 60,1 & $57,9-62,3$ & 9.725 \\
\hline Mato Grosso do Sul & 75,1 & $72,0-77,9$ & 712 & 77,2 & $74,0-80,0$ & 2.092 \\
\hline Mato Grosso & 72,9 & $69,2-76,4$ & 887 & 74,4 & $70,5-77,9$ & 2.554 \\
\hline Goiás & 58,6 & $54,5-72,6$ & 1.432 & 59,3 & $55,2-63,3$ & 4.168 \\
\hline Distrito Federal & 27,0 & $23,6-30,6$ & 280 & 30,2 & $26,5-34,2$ & 911 \\
\hline
\end{tabular}

Fonte: elaboração própria a partir dos dados da PNS 2019.

apenas $12,7 \%(\mathrm{RJ})$ e $4,1 \%(\mathrm{DF})$, respectivamente (Tabela 3 ). Os estados que tiveram reduções mais expressivas no recebimento de pelo menos uma visita domiciliar nos últimos doze meses foram AC, RO, ES, RJ, DF, PR, SC, com dez pontos ou mais de aumento da resposta não receberam VD nos últimos doze meses (Tabela 3).

Analisando-se a cobertura de USF por estrato de renda domiciliar per capita observa-se tendên- cia de coberturas superiores nos quintis mais baixos da renda per capita em gradiente decrescente conforme a renda sobe. Padrão similar ao que ocorria em 2013. Em 2019, a cobertura por USF é de $74,0 \%$ no primeiro quintil e de $38,1 \%$ no quintil 5, de domicílios de maior renda familiar per capita (Tabela 4a).

Analisando esta mesma cobertura estratificada pelo grau de escolaridade do responsável pelo 
Tabela 2. Evolução da proporção (\%) de pessoas moradoras em domicílios cadastrados em unidades de saúde da família, Brasil, Grandes Regiões, Unidades da Federação e situação do domicílio urbano/rural, 2013 e 2019. Valores de p, teste do Qui-quadrado para comparação entre os dois períodos.

\begin{tabular}{|c|c|c|c|c|c|}
\hline \multirow{2}{*}{$\begin{array}{l}\text { Brasil, Grandes Regiões e } \\
\text { Unidades da Federação }\end{array}$} & \multirow{2}{*}{2013} & \multirow{2}{*}{2019} & \multirow{2}{*}{$\begin{array}{l}\text { Variação } \\
\text { absoluta }\end{array}$} & \multirow{2}{*}{$\begin{array}{c}\text { Proportional } \\
\text { variation } \\
2019-2013\end{array}$} & \multirow{2}{*}{$\begin{array}{l}\chi^{2} \\
\mathbf{p}\end{array}$} \\
\hline & & & & & \\
\hline Brasil $^{*}$ & 56,1 & 62,6 & 6,5 & 11,6 & 0,0001 \\
\hline Urbano* $^{*}$ & 53,3 & 59,8 & 6,5 & 12,3 & $<0,0001$ \\
\hline Rural $^{*}$ & 72,2 & 78,9 & 6,7 & 9,2 & $<0,0001$ \\
\hline Norte $^{*}$ & 53,4 & 62,2 & 8,8 & 16,5 & 0,0001 \\
\hline Rondônia & 56,7 & 52,8 & $-3,9$ & $-6,9$ & 0,2538 \\
\hline Acre & 51,7 & 56,1 & 4,4 & 8,6 & 0,2246 \\
\hline Amazonas & 54,2 & 58,5 & 4,3 & 7,8 & 0,2311 \\
\hline Roraima & 55,0 & 54,0 & $-1,0$ & $-1,9$ & 0,7620 \\
\hline Pará $^{*}$ & 46,9 & 63,7 & 16,8 & 35,8 & $<0,0001$ \\
\hline Amapá* & 33,8 & 45,3 & 11,5 & 34,0 & 0,0149 \\
\hline Tocantins ${ }^{*}$ & 93,6 & 89,7 & $-3,9$ & $-4,2$ & 0,0088 \\
\hline Nordeste $^{\star}$ & 68,1 & 73,3 & 5,2 & 7,7 & $<0,0001$ \\
\hline Maranhão & 66,4 & 68,4 & 2,0 & 2,9 & 0,5359 \\
\hline Piauí ${ }^{*}$ & 80,2 & 90,7 & 10,5 & 13,0 & $<0,0001$ \\
\hline Ceará* & 67,6 & 73,8 & 6,2 & 9,2 & 0,0152 \\
\hline Rio Grande do Norte & 64,7 & 71,1 & 6,4 & 10,0 & 0,0546 \\
\hline Paraíba* & 81,0 & 86,7 & 5,7 & 7,0 & 0,0254 \\
\hline Pernambuco $^{\star}$ & 66,8 & 73,4 & 6,6 & 9,9 & 0,0171 \\
\hline Alagoas & 68,6 & 67,8 & $-0,8$ & $-1,1$ & 0,8476 \\
\hline Sergipe ${ }^{\star}$ & 72,7 & 82,6 & 9,9 & 13,6 & $<0,0001$ \\
\hline Bahia & 63,9 & 68,1 & 4,2 & 6,6 & 0,2012 \\
\hline Sudeste ${ }^{\star}$ & 48,2 & 54,6 & 6,4 & 13,2 & 0,0006 \\
\hline Minas Gerais & 72,2 & 73,0 & 0,8 & 1,2 & 0,7859 \\
\hline Espírito Santo & 57,3 & 61,7 & 4,4 & 7,6 & 0,2903 \\
\hline Rio de Janeiro* & 35,1 & 48,5 & 13,4 & 38,2 & $<0,0001$ \\
\hline São Paulo* & 41,2 & 47,7 & 6,5 & 15,7 & 0,0184 \\
\hline $\mathrm{Sul}^{\star}$ & 58,5 & 67,6 & 9,1 & 15,6 & 0,0001 \\
\hline Paraná* & 56,6 & 68,5 & 11,9 & 21,1 & 0,0017 \\
\hline Santa Catarina* & 76,5 & 87,2 & 10,7 & 14,0 & 0,0003 \\
\hline Rio Grande do Sul & 49,5 & 54,2 & 4,7 & 9,4 & 0,1997 \\
\hline Centro-Oeste ${ }^{\star}$ & 54,5 & 60,1 & 5,6 & 10,3 & 0,0122 \\
\hline Mato Grosso do Sul & 76,7 & 77,2 & 0,5 & 0,7 & 0,8317 \\
\hline Mato Grosso ${ }^{\star}$ & 66,0 & 74,4 & 8,4 & 12,8 & 0,0160 \\
\hline Goiás & 57,5 & 59,3 & 1,8 & 3,2 & 0,6363 \\
\hline Distrito Federal $^{\star}$ & 14,2 & 30,2 & 16,0 & 112,3 & $<0,0001$ \\
\hline Mato Grosso ${ }^{\star}$ & 66,0 & 74,4 & 8,4 & 12,8 & 0,0160 \\
\hline Goiás & 57,5 & 59,3 & 1,8 & 3,2 & 0,6363 \\
\hline Distrito Federal ${ }^{\star}$ & 14,2 & 30,2 & 16,0 & 112,3 & $<0,0001$ \\
\hline
\end{tabular}

Fonte: elaboração própria a partir dos dados da PNS 2019 e da PNS 2013.

domicílio que também pode ser considerado um proxy de renda, observa-se padrão similar: quanto mais baixo o nível de instrução, mais elevada a cobertura por USF. A cobertura de moradores em domicílios com responsável sem instrução, em 2019 foi de $76,9 \%$ e para aqueles com ensino superior completo foi 41,7\% (Tabela 4a).

Entre 2013 e 2019, observou-se aumento da cobertura em todos os quintis de renda, e em todos os níveis de escolaridade do responsável 
Tabela 3. Proporção de domicílios cadastrados em unidades de saúde da família há mais de um ano que receberam visita do ACS nos últimos doze meses, segundo a frequência. Brasil, Grandes Regiões, Unidades da Federação e situação do domicílio urbano/rural, 2013 e 2019.

\begin{tabular}{|c|c|c|c|c|c|c|c|c|c|c|c|}
\hline & \multirow{2}{*}{\multicolumn{2}{|c|}{ mensal }} & \multirow{2}{*}{\multicolumn{2}{|c|}{$\begin{array}{c}\text { a cada } 2 \\
\text { meses }\end{array}$}} & \multirow{2}{*}{\multicolumn{2}{|c|}{$\begin{array}{c}\text { de } 2 \text { a } 4 \\
\text { vezes }\end{array}$}} & \multirow{2}{*}{\multicolumn{2}{|c|}{ uma vez }} & \multirow{2}{*}{\multicolumn{2}{|c|}{$\begin{array}{c}\text { nenhuma } \\
\text { vez }\end{array}$}} & \multirow{3}{*}{$\begin{array}{c}\chi^{2} \\
\mathbf{p} \\
\text { Comparação } \\
\text { visita mensal }\end{array}$} \\
\hline & & & & & & & & & & & \\
\hline & 2013 & 2019 & 2013 & 2019 & 2013 & 2019 & 2013 & 2019 & 2013 & 2019 & \\
\hline Brasil $^{\star}$ & 47,2 & 38,4 & 11,1 & 10,5 & 13,4 & 16,1 & 10,6 & 11,2 & 17,7 & 23,8 & $<0,0001^{\star *}$ \\
\hline Urbano* & 43,6 & 34,4 & 11,1 & 10,5 & 14,1 & 16,7 & 11,4 & 12,0 & 19,8 & 26,3 & $<0,0001^{\star *}$ \\
\hline Rural $^{*}$ & 62,7 & 56,0 & 11,0 & 10,3 & 10,7 & 13,6 & 7,0 & 7,7 & 8,6 & 12,4 & $0,0002^{\star *}$ \\
\hline Norte ${ }^{*}$ & 49,6 & 38,7 & 14,9 & 12,2 & 13,1 & 17,5 & 11,6 & 12,7 & 10,8 & 19,0 & $<0,0001^{\star *}$ \\
\hline Rondônia* & 53,8 & 37,9 & 12,7 & 10,1 & 13,8 & 15,5 & 12,1 & 12,4 & 7,5 & 24,1 & $0,0002^{* *}$ \\
\hline Acre $^{\star}$ & 53,0 & 39,3 & 18,1 & 11,3 & 13,3 & 17,9 & 8,4 & 10,1 & 8,4 & 22,6 & $0,0006^{* *}$ \\
\hline Amazonas & 46,5 & 40,0 & 9,7 & 9,1 & 11,2 & 12,2 & 14,1 & 18,5 & 18,4 & 20,2 & 0,1208 \\
\hline Roraima* & 36,2 & 22,6 & 15,1 & 12,9 & 20,8 & 25,8 & 15,1 & 16,1 & 13,2 & 21,5 & $0,0001^{* *}$ \\
\hline Pará* & 41,6 & 35,4 & 18,0 & 12,0 & 15,6 & 21,0 & 13,1 & 11,4 & 11,7 & 20,2 & 0,0959 \\
\hline Amapá & 29,4 & 25,5 & 11,8 & 7,5 & 19,6 & 23,6 & 19,6 & 23,6 & 21,6 & 19,8 & 0,5764 \\
\hline Tocantins ${ }^{\star}$ & 70,8 & 53,2 & 14,6 & 18,6 & 7,4 & 11,5 & 4,6 & 7,7 & 2,6 & 9,2 & $<0,0001^{\star *}$ \\
\hline Nordeste $^{*}$ & 50,4 & 42,6 & 12,1 & 11,7 & 14,7 & 18,8 & 9,5 & 10,4 & 13,4 & 16,4 & $<0,0001^{\star *}$ \\
\hline Maranhão & 54,8 & 49,1 & 11,6 & 13,5 & 12,2 & 18,8 & 10,2 & 9,3 & 11,2 & 9,3 & 0,0792 \\
\hline Piauí ${ }^{*}$ & 53,3 & 39,8 & 14,2 & 11,1 & 11,9 & 20,6 & 12,1 & 11,4 & 8,6 & 17,1 & $0,0001^{* *}$ \\
\hline Ceará* & 49,4 & 41,0 & 10,9 & 8,9 & 15,4 & 17,6 & 10,1 & 10,7 & 14,2 & 21,8 & $0,0025^{\star *}$ \\
\hline Rio Grande do Norte & 43,3 & 35,8 & 12,1 & 14,1 & 14,8 & 21,5 & 11,8 & 11,4 & 18,1 & 17,3 & $0,0414^{* *}$ \\
\hline Paraíba ${ }^{*}$ & 61,4 & 52,6 & 13,3 & 10,1 & 11,4 & 15,3 & 8,5 & 11,0 & 5,4 & 11,2 & $0,0159^{* *}$ \\
\hline Pernambuco & 49,2 & 40,8 & 11,6 & 14,1 & 13,4 & 16,8 & 10,7 & 9,8 & 15,1 & 18,6 & $0,0052^{\star *}$ \\
\hline Alagoas & 52,9 & 58,0 & 12,5 & 11,6 & 14,1 & 13,9 & 6,2 & 6,6 & 14,3 & 9,9 & 0,1556 \\
\hline Sergipe & 42,6 & 37,9 & 14,3 & 10,5 & 17,1 & 22,3 & 9,6 & 9,2 & 16,6 & 20,2 & 0,1381 \\
\hline Bahia & 47,4 & 39,0 & 11,7 & 11,6 & 18,0 & 21,4 & 7,8 & 11,6 & 15,1 & 16,4 & 0,0551 \\
\hline Sudeste* & 41,5 & 33,4 & 9,6 & 9,3 & 13,6 & 15,8 & 11,4 & 11,5 & 23,9 & 30,0 & $0,0001^{\star *}$ \\
\hline Minas Gerais & 52,8 & 45,2 & 11,4 & 12,4 & 13,8 & 17,3 & 7,0 & 8,1 & 15,1 & 17,0 & $0,0449^{\star *}$ \\
\hline Espírito Santo ${ }^{\star}$ & 55,1 & 45,5 & 10,9 & 9,3 & 11,0 & 12,6 & 9,7 & 8,1 & 13,1 & 24,5 & $0,0301^{\star *}$ \\
\hline Rio de Janeiro* & 25,4 & 12,7 & 12,9 & 4,3 & 22,1 & 17,4 & 14,6 & 18,1 & 25,0 & 47,5 & $<0,0001^{\star *}$ \\
\hline São Paulo & 35,0 & 31,0 & 7,0 & 8,9 & 11,3 & 14,4 & 14,4 & 11,9 & 32,3 & 33,8 & 0,1868 \\
\hline $\mathrm{Sul}^{*}$ & 48,2 & 37,7 & 9,7 & 8,2 & 12,4 & 13,1 & 11,9 & 12,3 & 17,8 & 28,6 & $<0,0001^{\star \star}$ \\
\hline Paraná ${ }^{*}$ & 33,9 & 28,1 & 8,8 & 8,2 & 18,4 & 13,4 & 16,7 & 14,2 & 22,3 & 36,1 & 0,1305 \\
\hline Santa Catarina ${ }^{\star}$ & 65,1 & 52,1 & 10,6 & 7,8 & 8,8 & 9,2 & 6,6 & 9,4 & 8,8 & 21,5 & $0,0004^{\star *}$ \\
\hline Rio Grande do Sul & 47,8 & 34,2 & 9,8 & 8,8 & 9,4 & 16,7 & 11,7 & 13,1 & 21,3 & 27,2 & $0,0004^{\star \star}$ \\
\hline Centro-Oeste $^{*}$ & 58,3 & 46,8 & 13,7 & 14,4 & 9,6 & 11,4 & 7,5 & 9,6 & 10,8 & 17,8 & $<0,0001^{\star \star}$ \\
\hline Mato Grosso do Sul ${ }^{\star}$ & 62,0 & 54,3 & 15,9 & 13,6 & 9,7 & 11,2 & 5,5 & 10,0 & 6,6 & 10,9 & $0,0245^{\star *}$ \\
\hline Mato Grosso & 53,8 & 50,7 & 13,2 & 13,3 & 6,9 & 9,7 & 8,2 & 8,1 & 17,8 & 18,3 & 0,5011 \\
\hline Goiás* & 62,9 & 48,0 & 13,7 & 17,3 & 9,8 & 12,3 & 6,8 & 8,4 & 6,6 & 14,1 & $0,0003^{* *}$ \\
\hline Distrito Federal $^{*}$ & 9,1 & 4,1 & 4,7 & 4,1 & 24,7 & 13,4 & 24,7 & 21,2 & 36,7 & 57,2 & 0,0717 \\
\hline
\end{tabular}

(Tabela 4a). Destaca-se o expressivo aumento em $32 \%$ da cobertura de domicílios com responsável com ensino superior completo, que passou de $31,6 \%$ em 2013 para $41,7 \%$ em 2019, com quase duplicação de 7,4 milhões para 13,5 milhões de moradores cobertos, residentes em domicílios cujo responsável tem ensino superior completo (Tabela 4a).

Em todas as regiões do país se observa maior cobertura nos estratos de menor renda, com des- 
taque para a maior cobertura do primeiro quintil na região Nordeste com $81,4 \%$ dos moradores cobertos. Taxas mais homogêneas são observadas na região Sul com a cobertura mais elevada do quintil de maior renda: $52 \%$ e menor variação entre o primeiro e o quinto quintil com 19,7 pontos percentuais (entre $71,8 \%$ e $52,0 \%$ ), em contraste com o Nordeste onde a variação na cobertura de moradores segundo quintil de renda é de 43,2 pontos.

Visitas domiciliares mensais são também mais frequentes no estrato de população de menor renda (Tabela 4b). As visitas mensais apresentaram reduções em todos os estratos de renda entre 2013 e 2019, mas manteve-se gradiente com maior frequência de visita mensal $(44,4 \%)$ nos domicílios do quintil 1, mais pobres, e de menor frequência $(26,5 \%)$ no quintil 5 de maior renda. Coerentemente, a proporção de domicílios cadastrados que não receberam visita no último ano, é menor nos quintis de renda mais baixa: menos da metade no quintil 1 comparado ao quintil 5 (Tabela 4b).

Para conhecer a disponibilidade de uma fonte usual de cuidado, a PNS perguntou se o morador costuma procurar o mesmo lugar, mesmo médico ou mesmo serviço de saúde quando precisa de atendimento. A disponibilidade de um serviço de procura regular é maior entre os moradores cadastrados na USF, 78,7\% (IC95\%:78,0-79,4) do que entre os não cadastrados, 72,5\% (IC95\%: 71,4-73,7) e do que entre aqueles que não sabem a situação cadastral do domicílio (73,5\%; IC95\%: 72,0-75,0). Comparando-se com 2013, observase resultado similar para cadastrados que dispunham de um serviço de procura regular, 80,0\% (IC95\%: 79,0-81,0), e sua redução significativa $(\mathrm{p}=0,0046)$ entre não cadastrados na USF, que em 2013 era de 75,1\% (IC95\%: 73,9-76,3).

Os três serviços de procura regular mais frequentes dos moradores cadastrados na USF são: $56,1 \%$ UBS (posto ou centro de saúde ou USF), $16,0 \%$ consultórios particulares (ou clínica privada ou ambulatório de hospital privado), e 13,3\% serviços de pronto atendimento públicos (UPA, outro tipo de pronto-atendimento público 24 horas, pronto socorro ou emergência de hospital público) (Tabela 5). Entre a população não cadastrada na UBS, o principal serviço de procura regular são os consultórios particulares $(36,5 \%)$, seguidos pelas UBS $(28,1 \%)$ e pelos serviços de pronto atendimento públicos (16,0\%) (Tabela 5).

Os serviços de procura regular dos moradores residentes em domicílios cadastrados são, na grande maioria, públicos: $78,8 \%$, enquanto entre os não cadastrados esta proporção é de $52,2 \%$. Em 2013, estes percentuais eram $80,8 \%$ e $54,9 \%$, respectivamente (Tabela 5 ).

Tabela 4a. Proporção e número de moradores (por 1.000) em domicílios cadastrados em unidades de saúde da família, segundo o nível de instrução do responsável pelo domicílio e por quintil de renda familiar per capita. Brasil, PNS 2013 e 2019. Valores de p, teste do Qui-quadrado de Pearson ajustado para comparação entre os anos.

\begin{tabular}{|c|c|c|c|c|c|c|c|}
\hline & \multicolumn{3}{|c|}{2013} & \multicolumn{3}{|c|}{2019} & \multirow{2}{*}{$\begin{array}{l}\chi^{2} \\
\mathbf{p}\end{array}$} \\
\hline & $\%$ & IC 95\% & $\mathbf{n}$ & $\%$ & IC 95\% & $\mathbf{n}$ & \\
\hline \multicolumn{8}{|l|}{$\begin{array}{l}\text { Nível de instrução do responsável pelo } \\
\text { domicílio }\end{array}$} \\
\hline Sem instrução ou $<1$ a & 68,1 & $65,9-70,3$ & 13.592 & 76,9 & $75,1-78,6$ & 11.253 & $<0,0001$ \\
\hline Ensino fundamental incompleto & 64,2 & $62,5-65,8$ & 47.573 & 71,9 & $70,6-73,1$ & 49.512 & $<0,0001$ \\
\hline Ensino fundamental completo & 58,4 & $56,3-60,4$ & 16.505 & 64,5 & $62,7-66,1$ & 19.505 & $<0,0001$ \\
\hline Ensino médio completo & 49,9 & $48,2-51,6$ & 27.184 & 58,9 & $57,4-60,5$ & 3.751 & $<0,0001$ \\
\hline Superior completo & 31,6 & $29,4-33,9$ & 7.380 & 41,7 & $39,7-43,7$ & 13.503 & $<0,0001$ \\
\hline \multicolumn{8}{|c|}{ Quintis de renda per capita dos moradores } \\
\hline $1^{\circ}$ quintil & 67,2 & $65,3-69,0$ & 26.837 & 74,0 & $72,6-75,3$ & 38.674 & $<0,0001$ \\
\hline $2^{\circ}$ quintil & 63,6 & $61,7-65,5$ & 25.350 & 68,4 & $67,0-69,8$ & 34.224 & 0,0002 \\
\hline $3^{\circ}$ quintil & 60,7 & $58,9-62,5$ & 24.200 & 65,4 & $63,6-67,2$ & 23.694 & 0,0008 \\
\hline $4^{\circ}$ quintil & 54,1 & $52,1-56,1$ & 21.573 & 58,3 & $56,5-60,2$ & 21.701 & 0,0039 \\
\hline $5^{\circ}$ quintil & 34,8 & $32,9-36,9$ & 13.886 & 38,1 & $36,2-40,0$ & 12.833 & 0,0268 \\
\hline Total de moradores & 56,1 & $54,8-57,3$ & 111.883 & 62,6 & $61,5-63,7$ & 131.201 & \\
\hline
\end{tabular}


Tabela 4b. Proporção e número de domicílios (por 1.000) cadastrados em unidades de saúde da família, segundo a frequência de visitas domiciliares (mensal e nenhuma) e quintil de renda familiar per capita. Brasil, PNS 2013 e 2019.

\begin{tabular}{|c|c|c|c|c|c|c|c|}
\hline \multirow{2}{*}{ Visitas de ACS } & \multicolumn{3}{|c|}{2013} & \multicolumn{3}{|c|}{2019} & \multirow{2}{*}{$\begin{array}{l}\chi^{2} \\
\mathbf{p}\end{array}$} \\
\hline & $\%$ & CI 95\% & $\mathbf{N}$ & $\%$ & CI 95\% & $\mathbf{n}$ & \\
\hline \multicolumn{8}{|l|}{$\begin{array}{l}\text { Visitas mensais de ACS nos } \\
\text { últimos doze meses }\end{array}$} \\
\hline $1^{\circ}$ quintil & 53,1 & $50,7-55,5$ & 12.621 & 44,4 & $42,7-46,1$ & 15.753 & $<0,0001$ \\
\hline $2^{\circ}$ quintil & 51,4 & $48,9-53,8$ & 10.680 & 39,3 & $37,7-40,9$ & 12.003 & $<0,0001$ \\
\hline $3^{\circ}$ quintil & 48,1 & $46,0-50,2$ & 9.709 & 39,5 & $37,9-41,1$ & 8.252 & $<0,0001$ \\
\hline $4^{\circ}$ quintil & 44,1 & $41,7-46,7$ & 8.043 & 36,2 & $34,4-38,1$ & 7.137 & $<0,0001$ \\
\hline $5^{\circ}$ quintil & 38,1 & $35,3-40,9$ & 4.359 & 26,5 & $24,6-28,5$ & 3.058 & $<0,0001$ \\
\hline \multicolumn{8}{|c|}{ Nenhuma visita de ACS nos últimos doze meses ${ }^{\star}$} \\
\hline $1^{\circ}$ quintil & 12,4 & $11,0-14,0$ & 2.741 & 18,1 & $16,7-19,5$ & 6.231 & $<0,0001$ \\
\hline $2^{\circ}$ quintil & 14,2 & $12,7-15,8$ & 3.165 & 21,1 & $19,8-22,5$ & 6.553 & $<0,0001$ \\
\hline $3^{\circ}$ quintil & 17,3 & $15,6-19,1$ & 3.721 & 21,9 & $20,6-23,3$ & 4.993 & 0,0001 \\
\hline $4^{\circ}$ quintil & 20,2 & $18,3-22,3$ & 3.892 & 27,2 & $25,5-28,9$ & 5.404 & $<0,0001$ \\
\hline $5^{\circ}$ quintil & 25,6 & $23,1-28,2$ & 3.145 & 37,9 & $35,7-40,1$ & 4.398 & $<0,0001$ \\
\hline
\end{tabular}

Nota: Em 2013, as médias de renda familiar per capita para os quintis foram: $\mathrm{R} \$ 169,25$ (1); R $\$ 384,74$ (2); R \$617,51 (3); R \$976,10 (4); $\mathrm{R} \$ 3.035,79$ (5). Em 2019, R $\$ 275,91$ (1); $\mathrm{R} \$ 663,14$ (2); $\mathrm{R} \$ 1.042,72$ (3); $\mathrm{R} \$ 1.613,04$ (4) e $\mathrm{R} \$ 4.909,72$ (5). * Nos domicílios cadastrados há mais de doze meses.

Fonte: Elaboração própria, a partir dos dados da PNS 2019 e da PNS 2013.

Tabela 5. Serviço de procura regular por moradores (por 1.000) segundo cadastramento na USF e os tipos de serviços utilizados, 2013 e 2019.

\begin{tabular}{|c|c|c|c|c|c|c|c|c|c|c|c|c|}
\hline \multirow{3}{*}{$\begin{array}{l}\text { Tipo de serviço } \\
\text { utilizado }\end{array}$} & \multicolumn{6}{|c|}{2013} & \multicolumn{6}{|c|}{2019} \\
\hline & \multicolumn{3}{|c|}{ Com cadastro na USF } & \multicolumn{3}{|c|}{ Sem cadastro na USF } & \multicolumn{3}{|c|}{ Com cadastro na USF } & \multicolumn{3}{|c|}{ Sem cadastro na USF } \\
\hline & $\%$ & IC 95 & $\mathbf{n}$ & $\%$ & IC 95 & $\mathbf{n}$ & $\%$ & IC 95 & $\mathbf{n}$ & $\%$ & IC 95 & $\mathbf{n}$ \\
\hline $\begin{array}{l}\text { UBS, posto, centro } \\
\text { de saúde ou USF }\end{array}$ & 58,5 & $57,2-59,9$ & 52.419 & 32,0 & $30,2-33,8$ & 15.956 & 56,1 & $55,1-57,2$ & 58.000 & 28,1 & $26,3-30,0$ & 11.270 \\
\hline $\begin{array}{l}\text { Consultório } \\
\text { particular }{ }^{a}\end{array}$ & 13,2 & $12,5-14,0$ & 11.811 & 32,9 & $31,2-34,6$ & 16.415 & 16,0 & $15,4-16,7$ & 16.562 & 36,5 & $34,7-38,3$ & 14.626 \\
\hline $\begin{array}{l}\text { Pronto atendimento } \\
\text { público }^{\mathrm{b}}\end{array}$ & 9,9 & $9,0-10,9$ & 8.861 & 12,9 & $11,8-14,0$ & 6.413 & 13,3 & $12,4-14,2$ & 13.741 & 16,0 & $14,7-17,4$ & 6.404 \\
\hline $\begin{array}{l}\text { Ambulatório } \\
\text { hospital público }\end{array}$ & 10,6 & $9,8-11,4$ & 9.448 & 8,3 & $7,5-9,1$ & 4.139 & 6,8 & $6,4-7,3$ & 7.047 & 5,2 & $4,4-6,1$ & 2.077 \\
\hline $\begin{array}{l}\text { PA ou emergência } \\
\text { hospital privado }\end{array}$ & 2,8 & $2,4-3,2$ & 2.470 & 8,6 & $7,7-9,6$ & 4.282 & 2,6 & $2,3-2,9$ & 2.637 & 8,0 & $7,0-9,3$ & 3.220 \\
\hline Farmácia & 2,9 & $2,6-3,3$ & 2.619 & 2,9 & $2,5-3,3$ & 1.429 & 2,2 & $1,9-2,4$ & 2.227 & 2,6 & $2,2-3,1$ & 1.047 \\
\hline $\begin{array}{l}\text { Policlínica, PAM } \\
\text { ou Centro de } \\
\text { Especialidades } \\
\text { público }\end{array}$ & 1,8 & $1,5-2,1$ & 1.580 & 1,8 & $1,4-2,2$ & 887 & 2,6 & $2,2-3,0$ & 2.640 & 2,9 & $2,4-3,5$ & 1.172 \\
\hline $\begin{array}{l}\text { Atendimento } \\
\text { domiciliar }\end{array}$ & 0,1 & $0,0-0,2$ & 124 & 0,4 & $0,2-0,7$ & 186 & 0,2 & $0,1-0,2$ & 161 & 0,1 & $0,1-0,2$ & 56 \\
\hline Outro serviço & 0,2 & $0,2-0,3$ & 219 & 0,4 & $0,3-0,6$ & 218 & 0,3 & $0,2-0,4$ & 289 & 0,5 & $0,3-0,8$ & 206 \\
\hline
\end{tabular}


Comparando-se os resultados de 2019 com 2013, observa-se que a UBS foi mencionada como serviço de procura regular por $56,1 \%$ (55,1\%-57,2\%) dos moradores cadastrados em 2019 , enquanto este percentual era de 58,5\% (57,2\%-59,9\%) em 2013, com diferença significativa $(\mathrm{p}=0,0092)$. Por outro lado, observa-se um aumento significativo na procura dos serviços de pronto atendimento públicos como fonte regular de cuidado que passam de 9,9\% para 13,3\% $(\mathrm{p}<0,0001)$ entre os cadastrados e de $12,9 \%$ para $16,0 \%(\mathrm{p}=0,0005)$ entre os não cadastrados (Tabela 5$)$.

\section{Discussão}

Os resultados da PNS 2019 ratificam que a ESF é a modalidade predominante da APS no SUS, chegando a $62,6 \%$ dos brasileiros em 2019. Os percentuais de cobertura observados nas regiões Nordeste e Sul, são maiores, mantendo o padrão da PNS de 2013. No entanto, é no Sudeste, que vive a maior parte dos brasileiros cadastrados a uma USF, espelhando o padrão de distribuição populacional nacional.

A cobertura é mais elevada entre a população mais vulnerável, seja considerando a escolaridade do chefe da família ou a renda familiar per capita dos moradores. Sua presença é mais significativa nas áreas rurais onde, no geral, vivem populações com piores condições de vida e maiores dificuldades de acesso aos serviços de saúde, localidades nas quais os serviços privados são residuais e o SUS é o responsável pela atenção à saúde. Nesse sentido, a ESF alcança com maior intensidade as populações mais vulneráveis.

Evidencia-se aceleração da expansão da cobertura populacional da ESF entre 2013 e 2019, comparando-se com o período 2008-2013. Nos seis anos entre 2013 e 2019, a cobertura aumentou 6,5 pontos percentuais: uma média de aumento de 1,1 pontos ao ano, incluindo mais 18,7 milhões de pessoas. Entre 2008 e 2013, a cobertura aumentara em 2,5 pontos percentuais, de $50,9 \%$ para $53,4 \%{ }^{6}$; isto é 0,5 pontos ao ano.

A aceleração da expansão da ESF pode estar relacionada ao sucesso do provimento de médicos pelo Programa Mais Médicos (PMM) instituído pelo governo Dilma a partir de 2013 que contribuiu para implantação de mais 8,8 mil equipes ESF, entre setembro de 2013 (34.892), início da chegada dos médicos e médicas PMM aos municípios e outubro de 2018 (43.735), último mês de atuação dos médicos cubanos, conforme o Eges- tor $\mathrm{AB}^{14}$. Implantado predominantemente em municípios com maior vulnerabilidade ou periferias das metrópoles, diversos estudos mostram melhoria da cobertura e da assistência, e redução da escassez de médicos a partir do $\mathrm{PMM}^{15-17}$. Ainda que tenha ocorrido inserção de médicos PMM em equipes existentes com presença intermitente deste profissional, reduzindo rotatividade, já entre 2013 e 2015, foram implantadas mais sete mil novas equipes ${ }^{10}$ contribuindo sobremaneira para a expansão da cobertura da ESF, uma vez que médicos PMM somente podiam ser inseridos em equipes da ESF. Com o desmantelamento do PMM em 2019, e a proposta de substituição pelo Médicos pelo Brasil, a ser implantado por uma agência privada ${ }^{18}$, é de se supor que esta evolução positiva seja interrompida.

Chama também a atenção que em 2019, proporção similar de respondentes a 2013 (11\%), permaneciam sem saber responder se o domicílio estava cadastrado ou não. Esta dúvida sugere pouco conhecimento do informante sobre a Estratégia Saúde da Família e a necessidade de melhor comunicação.

Os resultados da PNS 2019 reiteram que a Saúde da Família se mantém como política equitativa na medida em que a cobertura é maior entre os mais pobres, reduzindo as diferenças socioeconômicas ao acesso a serviços de saúde de APS $^{19}$ com resultados consistentes, seja em relação à renda ou à escolaridade, entendidos como proxys de vulnerabilidade. Os efeitos da ESF na redução das desigualdades são descritos em farta bibliografia, nas mais diversas dimensões, entre estas as inequidades raciais na mortalidade por causas preveníveis ${ }^{20}$.

Um dos resultados que mais chama a atenção é a diminuição da proporção de população cadastrada que recebe visita mensal do ACS, ainda que em termos absolutos este contingente populacional tenha se ampliado. Esta redução pode estar associada a diversos fatores. Por um lado, uma expansão acentuada de cobertura no Rio de Janeiro, especialmente na capital, e no Distrito Federal, locais onde o modelo assistencial, de certa forma legitimados pela PNAB 2017, priorizou a centralidade no cuidado individual com redução acentuada do número de $\mathrm{ACS}^{21,22}$. Por outro lado, nos estados que já apresentavam maior cobertura, a ampliação se espraia por territórios de moradia de classe média, que apresentam dificuldades distintas para efetivação da visita domiciliar como ausência dos moradores por trabalho fora, edifícios e condomínios que dificultam a entrada de ACS, resistência dos moradores à $\mathrm{VD}$, entre outras. 
Outros fatores que podem estar associados são mudanças observadas nas responsabilidades dos ACS com intensificação de atividades dentro das UBS, no acolhimento, no preenchimento de formulários do Bolsa Família e dos sistemas de informação, ou mesmo fora da UBS como a entrega de referências de exames e consultas especializadas agendadas. Estas atividades refletem um escopo de práticas com maior dedicação a atividades administrativas, de apoio, burocratizadas, em detrimento do tempo para visitas domiciliares e atuação no território ${ }^{23,24}$. Morosini e Fonseca ${ }^{24}$ alertam para a gradual alteração de sentido do trabalho do ACS no território, de um escopo complexo e de ação comunitária, na produção do diagnóstico da comunidade, para tarefas operacionais como apenas cadastrar e manter os cadastros atualizados. Além disso, as reais possibilidades de privatização e precificação das ações da APS a partir da ADAPS e carteira de serviços ${ }^{25}$ pode ser mais um elemento a enfraquecer a dimensão comunitária da ESF, como já se observa em contextos de privatização da gestão via OSS ${ }^{22}$.

Problemas na organização do processo de trabalho do ACS foram também evidenciados em estudo transversal realizado em 2015 com amostra nacional representativa realizado por Nunes et al apontando centralidade das visitas a determinados grupos, em necessidades individuais e visita domiciliar realizada com frequência mensal por somente $67 \%$ do $\mathrm{ACS}^{26}$.

Reconhece-se que o modelo assistencial da ESF centrado na vigilância em saúde com orientação comunitária apresenta limites em sua implementação. Não obstante, alerta-se para os potenciais efeitos negativos da reorientação do modelo assistencial, impulsionada pela Política Nacional de Atenção Básica de 2017 e por uma série de iniciativas a partir de 2019, que levam ao desiquilíbrio entre o cuidado individual e coletivo, com ênfase no pronto-atendimento. Ao mesmo tempo que a PNAB 2017 permitiu estabelecer equipes SF com apenas um ACS e equipes de atenção básica sem ACS, a Portaria 2979 de 2019 equiparou o financiamento das equipes de atenção básica e equipes SF, abolindo a prioridade para a ESF, enfraquecendo a dimensão comunitária e coletiva da APS no SUS. A continuidade destas políticas aponta para uma ainda maior redução das visitas domiciliares dos ACS ao longo do tempo.

$\mathrm{O}$ acesso aos cuidados de saúde é um conceito complexo e multidimensional ${ }^{27}$. Dispor de uma fonte usual de cuidado é um clássico indicador de acesso e de disponibilidade de serviços. Temse demostrado, desde longa data, que pessoas que dispõem de uma fonte usual de cuidado recebem mais diversos serviços preventivos e terapêuticos para enfermidades crônicas ${ }^{28-30}$. Na PNS este indicador é investigado como a disponibilidade de um serviço de saúde de procura regular.

Um dos atributos da APS é ser o serviço de primeiro contato, porta de entrada do sistema. Um requisito para cumprir este atributo e proporcionar longitudinalidade é que a UBS se constitua na fonte usual de cuidado. Desde 1998, com a então nova modalidade de financiamento (Piso de Atenção Básica) com repasses fundo a fundo que promoveu a capilarização da APS no país, as pesquisas de base populacional (PNAD-Saúde e PNS) vem mostrando melhora neste indicador, com proporções crescentes de moradores dispondo de um serviço de procura regular ${ }^{31}$.

Embora a UBS permaneça como principal serviço de busca regular, para 69 milhões de moradores (cadastrados e não cadastrados), percebe-se aumento da população que busca serviços de pronto-atendimento público, o que pode indicar competição pela porta de entrada entre o modelo assistencial da atenção básica e as unidades de pronto atendimento (UPA). No país, ao final dos anos 2000, ocorreu indução federal para implantação das unidades de pronto atendimento (UPA) no âmbito da política nacional de atenção às urgências. Em decorrência, houve grande expansão destas unidades, sob gestão municipal, que não lograram, contudo, a integração nas RAS, ocupando espaços de insuficiência quantitativa e qualitativa da APS e da atenção hospitalar ${ }^{32,33}$.

\section{Considerações finais}

Os resultados da PNS 2019 ratificam que a ESF se mantém como o principal modelo de APS no SUS, que apresentou resultados exitosos ao longo do tempo com sua orientação comunitária e para a equidade. A direcionalidade da política nacional recente vem promovendo expressiva ruptura em relação à prioridade do incentivo para a ESF, sem, contudo, apresentar respostas e propostas aos problemas históricos para qualificação da APS no país. As possibilidades de ausência do ACS na equipe afetam um dos pilares do modelo assistencial que caracteriza a ESF em seu componente comunitário e de promoção da saúde, pautado pela concepção da determinação social do processo saúde-doença e da clínica ampliada ${ }^{25}$. 
Nesse cenário, a continuação de pesquisas de base populacional como a PNS é imprescindível para o monitoramento e aperfeiçoamento da política de saúde no país, para promoção de comparações internacionais e, sobretudo, para o acompanhamento atento por todos os atores implicados na defesa do direito à saúde, integral, pública e universal.

Para finalizar é imperativo destacar a importância e potencialidade da Estratégia Saúde da Família com sua capilaridade, cobertura de 131 milhões de brasileiros e seus atributos de respon- sabilidade territorial e orientação comunitária no enfrentamento da pandemia de Covid-19: na vigilância em saúde com detecção, notificação, rastreamento e acompanhamento dos casos e contatos em isolamento domiciliar; na comunicação e educação em saúde; no cuidado de casos leves e moderados; no apoio social às populações vulneráveis e grupos de risco em articulação com organizações e lideranças locais; e na continuidade do cuidados ${ }^{34}$ deste enorme contingente populacional que tem a UBS como seu serviço de procura regular.

\section{Colaboradores}

L Giovanella e A Bousquat trabalharam na concepção, metodologia, análise de dados e redação final. PF Almeida trabalhou nas análises, discussão e redação final. S Schenkman trabalhou na metodologia e análise dos dados. LMV Sardinha e MLFP Vieira trabalharam na concepção, gestão e desenvolvimento da pesquisa e na revisão final do artigo. 


\section{Referências}

1. Hansen J, Groenewegen PP, Boerma WG, Kringos DS. Living in a country with a strong primary care system is beneficial to people with chronic conditions. Health Aff (Millwood) 2015; 34(9):1531-1537.

2. Castro MC, Massuda A, Almeida G, Menezes-Filho NA, Andrade MV, Noronha KVMS, Rocha R, Macinko J, Hone T, Tasca R, Giovanella L, Malik AM, Werneck H, Fachini LA, Atun R. Brazil's unified health system: the first 30 years and prospects for the future. Lancet 2019; 394(10195):345-356.

3. Rasella D, Harhay MO, Pamponet ML, Aquino R, Barreto ML. Impact of primary health care on mortality from heart and cerebrovascular diseases in Brazil: a nationwide analysis of longitudinal data. BMJ 2014; 349:g4014.

4. Pinto LF, Giovanella L. The Family Health Strategy: expanding access and reducing hospitalizations due to ambulatory care sensitive conditions (ACSC) Cien Saude Colet 2018; 23(6):1903-1914.

5. Starfield B. Atenção Primária: equilíbrio entre necessidades de saúde, serviços e tecnologia. Brasília: MS; 2002

6. Malta DC, Santos MAS, Stopa SR, Vieira JEB, Melo EA, Reis AAC. A Cobertura da Estratégia de Saúde da Família (ESF) no Brasil, segundo a Pesquisa Nacional de Saúde, 2013. Cien Saude Colet 2016; 21(2):327-338.

7. Stopa SR, Malta DC, Monteiro CN, Szwarcwald CL, Goldbaum M, Cesar CLG. Acesso e uso de serviços de saúde pela população brasileira, Pesquisa Nacional de Saúde 2013. Rev Saude Pubical 2017; 51(Supl.1):3s.

8. Machado CV, Lima LD, Baptista TWF. Políticas de saúde no Brasil em tempos contraditórios: caminhos e tropeços na construção de um sistema universal. Cad Saude Publica 2017; 33(Supl.2):e00129616.

9. Báscolo E, Houghton N, Del Riego A. Leveraging household survey data to measure barriers to health services access in the Americas. Rev Panam Salud Publica 2020; 44:e100.

10. Giovanella L, Mendonça MHM, Fausto MCR, Almeida PF, Bousquat A, Lima JG, Seidl H, Franco CM, Fusaro ER, Almeida SZF. A provisão emergencial de médicos pelo Programa Mais Médicos e a qualidade da estrutura das unidades básicas de saúde. Cien Saude Colet 2016; 21(9):2697-2708.

11. Morosini MVGC, Fonseca AF, Baptista TWF. Previne Brasil, Agência de Desenvolvimento da Atenção Primária e Carteira de Serviços: radicalização da política de privatização da atenção básica? Cad Saude Publica 2020; 36(9):e00040220.

12. Instituto Brasileiro de Geografia e Estatística (IBGE). Pesquisa nacional de saúde 2019. Informações sobre domicílios, acesso e utilização dos serviços de saúde: Brasil, grandes regiões e unidades da federação. Rio de Janeiro: IBGE; 2020

13. Stopa SR, Szwarcwald CL, Oliveira MM, Gouvea ECDP, Vieira MLFP, Freitas MPS. Pesquisa Nacional de Saúde 2019: histórico, métodos e perspectivas. Epidemiol Serv Saude 2020; 29(5):e2020315.

14. Brasil. Ministério da Saúde (MS). E-Gestor Atenção Básica: espaço para informação e acesso aos sistemas de Atenção Básica [Internet]. 2017 [acessado 2010 Out 26]. Disponível em: https://egestorab.saude.gov.br/ paginas/acessoPublico/relatorios/relHistoricoCoberturaAB.xhtml
15. Medina MG, Almeida PFL Juliana G, Moura D, Giovanella L. Programa Mais Médicos: mapeamento e análise da produção acadêmica no período 2013-2016 no Brasil. Saúde Debate 2018; 42(n. esp. 1):346-360.

16. Girardi SN, Van Stralen ACS, Cella JN, Wan Der Maas L, Carvalho CL, Faria EO. Impacto do Programa Mais Médicos na redução da escassez de médicos em Atenção Primária à Saúde. Cien Saude Colet 2016; 21(9):2675-2684.

17. Oliveira JPA, Sanchez MN, Santos LMP. O Programa Mais Médicos: provimento de médicos em municípios brasileiros prioritários entre 2013 e 2014. Cien Saude Colet 2016; 21(9):2719-2727.

18. Giovanella L, Bousquat A, Almeida PF, Melo EA, Medina MG, Aquino R, Mendonça MHM. Médicos pelo Brasil: caminho para a privatização da atenção primária à saúde no Sistema Único de Saúde? Cad Saude Publica 2019; 35(10):e00178619.

19. Andrade, MV, Noronha K, Barbosa ACQ, Rocha TAH, Silva NB, Calazans JÁ, Souza MN, Carvalho LR, Souza A. A equidade na cobertura da Estratégia Saúde da Família em Minas Gerais, Brasil. Cad Saude Publica 2015; 31(6):1175-1187.

20. Hone T, Rasella D, Barreto ML, Majeed A, Millett C. Association between expansion of primary healthcare and racial inequalities in mortality amenable to primary care in Brazil: A national longitudinal analysis. PLoS Med 2017; 14(5):e1002306.

21. Bousquat A, Barros NF, Gomes L. Brasília saudável: a estratégia de saúde da família e a conversão do modelo assistencial [relatório de pesquisa]. Rede de Pesquisa em APS: OPAS; 2019.

22. Melo EA, Mendonça MHM, Teixeira M. A crise econômica e a atenção primária à saúde no SUS da cidade do Rio de Janeiro, Brasil. Cien Saude Colet 2019; 24(12):4593-4598.

23. Nogueira ML Expressões da precarização no trabalho do agente comunitário de saúde: burocratização e estranhamento do trabalho. Saude Soc 2019; 28(3):309323.

24. Morosini MV, Fonseca AF. Os agentes comunitários na Atenção Primária à Saúde no Brasil: inventário de conquistas e desafios. Saúde Debate 2018; 42(n. esp. 1):261-274.

25. Giovanella L, Franco CM, Almeida PF. Política Nacional de Atenção Básica: para onde vamos?. Cien. Saude Colet 2020; 25(4):1475-1482.

26. Nunes CA, Aquino R, Medina MG, Vilasbôas ALQ, Pinto Júnior EP, Luz LA. Visitas domiciliares no Brasil: características da atividade basilar dos Agentes Comunitários de Saúde. Saúde Debate 2018; 42(n. esp. 2):127-144.

27. Travassos C, Martins M. Uma revisão sobre os conceitos de acesso e utilização de serviços de saúde. Cad Saude Publica 2004; 20 (Supl. 2):S190-S198.

28. Ettner SL. The timing of preventive services for women and children: the effect of having a usual source of care. Am J Public Health 1996; 86(12):1748-1754.

29. Mark TL, Paramore LC. Pneumococcal pneumonia and influenza vaccination: access to and use by U.S. Hispanic Medicare beneficiaries. Am J Public Health 1996; 86(11):1545-1550. 
30. Moy E, Bartman BA, Weir MR. Access to hypertensive care: effects of income, insurance, and source of care. Arch Intern Med 1995; 155(14):1497-1502.

31. Giovanella L, Mendonça MHM. Atenção primária à saúde. In: Giovanella L, Escorel S, Lobato LVC, Noronha JC, Carvalho AI, organizadores. Políticas e sistema de saúde no Brasil. $2^{\text {a }}$ ed. Rio de Janeiro: Editora Fiocruz; 2012. p. 493-546.

32. Konder MT, O'Dwyer G. As Unidades de Pronto-Atendimento na Política Nacional de Atenção às Urgências. Physis 2015; 25(2):525-545.

33. O'Dwyer G, Konder MT, Reciputti LP, Lopes MGM, Agostinho DF, Alves GF. O processo de implantação das unidades de pronto atendimento no Brasil. Rev Saude Publica 2017; 51:125.

34. Medina MG, Giovanella L, Bousquat A, Mendonça MHM, Aquino R. Atenção primária à saúde em tempos de COVID-19: o que fazer? Cad Saude Publica 2020; 36(8):e00149720.

Artigo apresentado em 12/11/2020

Aprovado em 03/12/2020

Versão final apresentada em 05/12/2020

Editores-chefes: Maria Cecília de Souza Minayo, Romeu Gomes, Antônio Augusto Moura da Silva 